\title{
Brain Correlates of Lane Changing Reaction Time in Simulated Driving
}

\author{
Huaijian Zhang ${ }^{\dagger}$, Ricardo Chavarriaga ${ }^{\dagger}$, Lucian Gheorghe ${ }^{\dagger, \ddagger}$, José del R. Millán \\ $\uparrow$ Chair on Non-Invasive Brain-Machine Interface (CNBI), Center for Neuroprosthetics, \\ Ecole Polytechnique Fédérale de Lausanne, Switzerland \\ ‡ Mobility Services Laboratory, Nissan Research Center, Nissan Motor Co., Japan \\ \{huaijian.zhang, ricardo.chavarriaga, lucian.gheorghe, jose.millan\}@epfl.ch
}

\begin{abstract}
Psychophysical studies have reported correlation between neural activity in frontal and parietal areas and subject's reaction time in simple tasks. Here we study whether similar correlates can also be identified in driver's electroencephalography (EEG) activity when they perform steering actions triggered by exogenous stimuli (e.g. obstacles along the road). We report analysis of the EEG signals of fifteen subjects while they drive in a realistic car simulator. We found that the peak latency of the event-related potentials in frontal and parietal areas significantly correlates with the onset of the steering behavior. Similarly, modulations of the power in the theta band $(4-8 \mathrm{~Hz})$ prior to the action also correlates with the reaction times. These results provide evidence of reliable neural markers of the driver's response variability.

Index Terms-Scalp EEG, driving task, reaction time, stimulus-driven reaction and event-related potential.
\end{abstract}

\section{INTRODUCTION}

Reaction time measures how fast people can respond to the presentation of a sensory stimulus and reflects the functionality of the central nervous system [1]. The reaction time depends on the characteristics of the stimulus and subject's mental states. The task urgency is one of the important characteristics that affect the reaction time, which is correlated with the contextual environment. The translation between the task urgency and the motor reaction is modulated by afferent and cortical systems [2]. Recent studies have shown evidence of a correlation between brain activities and reaction time, particularly in frontal and parietal areas [3], [4]. Furthermore, the latter region is believed to be activated earlier than frontal areas during stimulus-driven behaviors [5]. However these studies have mainly been performed using psychophysical protocols in well controlled situations, leaving open the question whether the same correlates also appear in more complex tasks.

At the same time, the analysis of the brain activity generated during driving has gained increased attention in recent years. This activity reflects underlying cognitive processes and can potentially be exploited to improve driving assistance systems for intelligent cars [6], [7], [8]. For example, recent studies have focused on detecting anticipated and emergency braking [7], [9], steering actions [8] as well as workload and levels of attention [10].

The present study investigates the neural correlates of reaction time in a driving task. We analyzed electroencepha- lography (EEG) event-related potentials (ERP) and spectral modulations elicited by the appearance of obstacles that trigger lane changes and their relation to the steering reaction. In particular we focus on identifying reliable brain activity markers of the response variability. Their correlation with the responding speed provides evidence linking the reaction time to specific brain patterns. These reported cortical patterns contribute to improve our understanding of the neural basis of stimulus driven behavior during car driving.

\section{Methods}

\section{A. Experimental protocols}

Fifteen subjects (three females, mean age 26.83 \pm 3.04 ) participated in the experiments. They were all $\mathrm{MSc}$ or $\mathrm{PhD}$ students and had normal or corrected-to-normal vision. All subjects had a valid driving license. None of them reported any known neurological or psychiatric disease. The experimental protocols were approved by the research ethical committee of the EPFL Brain and Mind institute and all participants gave their informed consent.

In the experiment, subjects seated in a car simulator and were asked to drive at high speed (at about $95 \mathrm{KMH}$ ) along a virtual 2-lane road. There were no other cars on the road. At any given moment an obstacle blocking one of the lanes could appear in front of the car, see Figure 1. If the obstacle was in the same lane, the subject was required to steer the car to the other lane to avoid the collision; otherwise, the subject should remain in the same lane. The probability of the obstacle to be in the same lane as the car was $25 \%$. The distance between the vehicle and the obstacle at the moment of its appearance was variable in order to study different types of reaction, ranging from rapid responses when the obstacle appears at close distance to self-paced, slower responses when it is far ahead. Given the protocol drivers were expected to maintain high levels of vigilance during the experiment.

To allow subjects to successfully avoid collisions throughout the experiment, the distance between the car and the obstacle at its appearance was chosen randomly from an uniform distribution between $40 \mathrm{~m}$ and $70 \mathrm{~m}$. The inter-trial interval (i.e. time between the appearance of two consecutive obstacles) was at least $5 \mathrm{sec}$ (corresponding to a distance of 

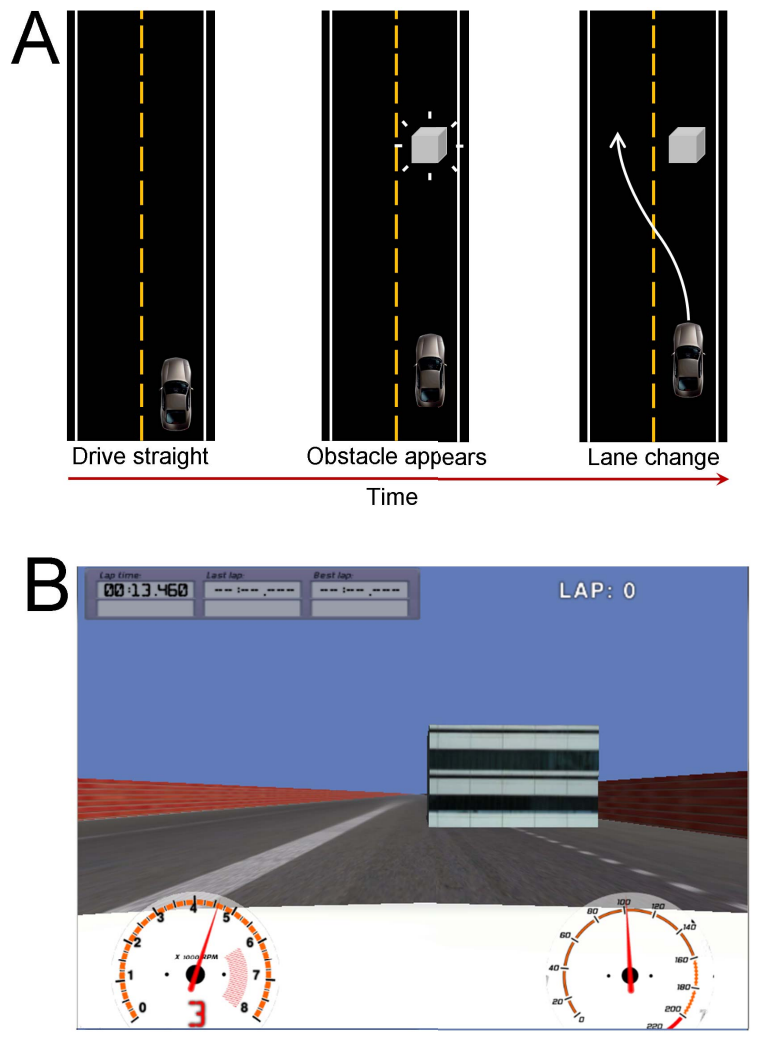

Fig. 1. Experimental protocol. A. Timing of the protocol. Subject drives straight in a two lane motor way. An obstacle may appear either in the same lane (rare condition, $25 \%$ ) or the other lane (frequent condition, $75 \%$ ). The subject needs to change lane immediately if the obstacle is in the same lane to avoid collision. B. The screenshot of the 3D environment as presented to the subject in the car simulator.

$150 \mathrm{~m})$. Therefore there was no overlapping between consecutive trials.

Each subject performed six runs in a single recording session, where each of them comprised the appearance of 87 obstacles (i.e. trials). The duration of one run was about 9 minutes and 30 seconds, resulting in a total recording time of about one hour. Overall, we obtained 522 trials per subject yielding $137.6 \pm 15.2$ trials where a lane change was required. Trials in which the subject steered during the second before the appearance of the obstacle were removed from the analysis. Since subjects may have different number of trials, we uniformly selected (according to the occurrence order) 100 trials for each subject and ordered them by their reaction time (i.e. rank 1 will correspond to the fastest trial, while rank 100 will be the slowest one). We averaged trials having the same rank -yielding 100 ERPs, one for each ranked speed- and computed the correlation between the EEG features and the speed of response.

\section{B. Experimental setup}

The experimental set-up consisted of a realistic car simulator as shown in Figure 2. Car related data (e.g. steering,

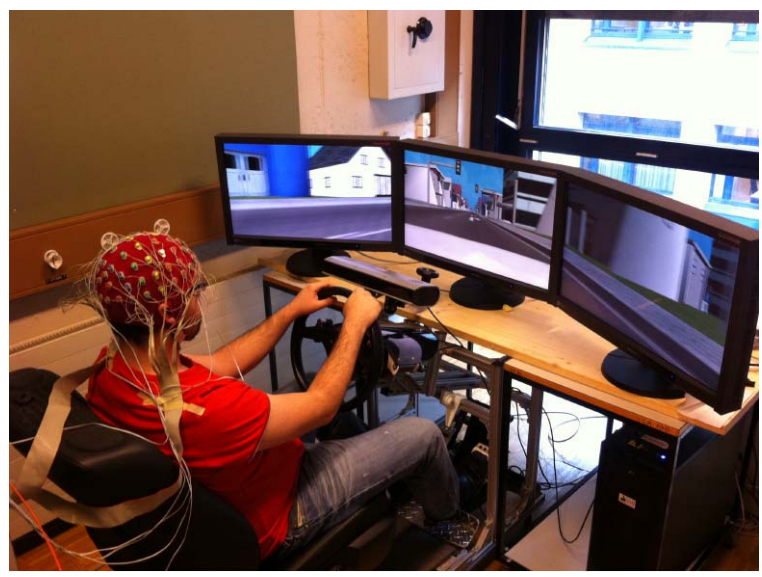

Fig. 2. Experimental protocol. Realistic car simulator including steering wheel, car pedals and three 3D monitors showing the virtual road environment. The subject wearing a 64-channel EEG cap can also be observed.

braking, accelerating and location) from the driving simulator was logged at $256 \mathrm{~Hz}$. The simulated driving environment was built using the software Blender (http://www.blender.org) and presented using a customized open source racing program (Vdrift) on three 27" 3D monitors. These monitors do not require the subject, who were seated at about $120 \mathrm{~cm}$ from the screens, to wear 3D glasses.

\section{Data recording and processing}

The driver's reaction time was estimated as the time it took for the steering values to exceed a threshold within $1 \mathrm{~s}$ after the obstacle appearance. The threshold was determined empirically. In this analysis we used the absolute value of steering data to define lane changes, thus the direction of the change (i.e. left-to-right or right-to-left) was not taken into account. In our experimental setup, the values of the steering data ranged from 0.0 to 0.3 (a.u.) corresponding to the range from straight driving to sharp steering turns.

Scalp EEG was recorded from 64 electrodes (Biosemi Active Two, The Netherlands) with an extended 10-20 system montage at a sampling rate of $2048 \mathrm{~Hz}$, which was downsampled to $512 \mathrm{~Hz}$ after recording. The most peripheral electrodes were discarded to reduce the influence of artifact contamination, yielding a total of 41 channels kept for further analysis. EEG and car-related recordings were synchronized for off-line analysis using an event signal sent from the driving simulator to the EEG recording device via parallel port.

For analysing ERPs, we filtered the EEG data in the frequency band $\left[\begin{array}{ll}1 & 10\end{array}\right] \mathrm{Hz}$ with a $4^{\text {th }}$ order non-causal Butterworth filter. The spectral analysis was performed in the range [1 50] Hz. Common average reference was used as spatial filter. Power spectral density (PSD) of the single trials was computed by 1024-point discrete Fourier transform with a sliding Hamming window of $250 \mathrm{~ms}$, and $218.75 \mathrm{~ms}$ overlapping was used in order to balance the smoothness and resolution in the time domain. For analysing ERPs and PSDs, EEG was segmented into epochs spanning from $-1 \mathrm{~s}$ to $1.5 \mathrm{~s}$ 

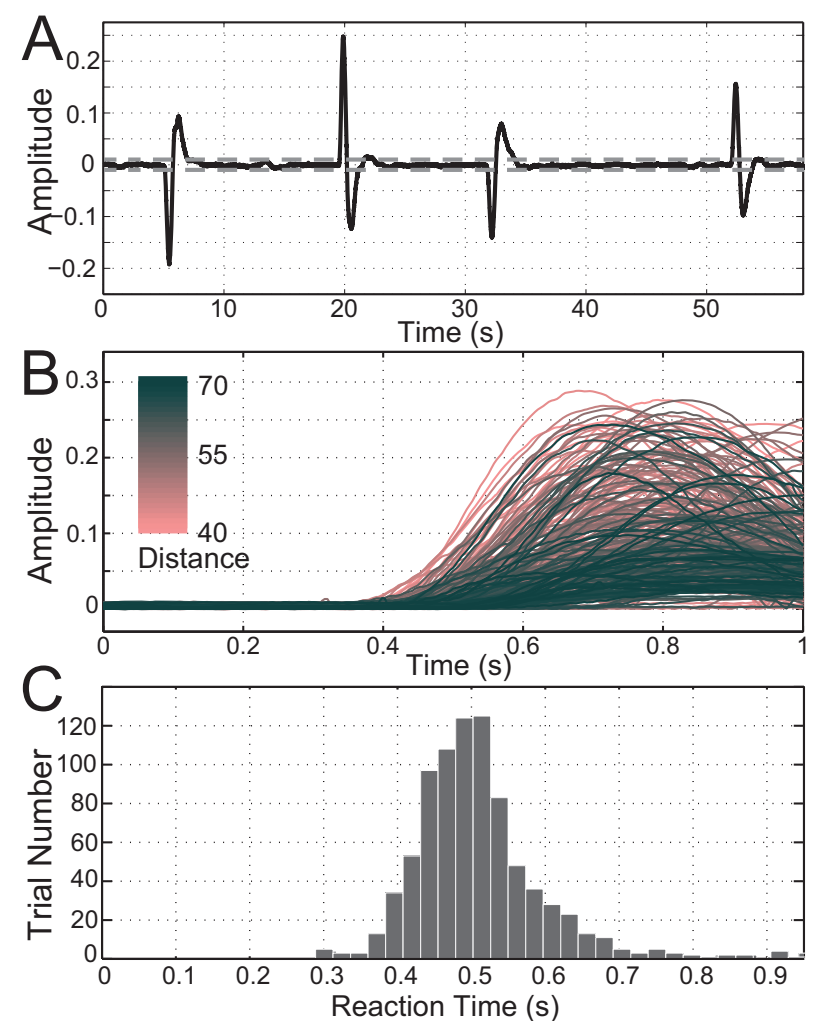

Fig. 3. Steering behavior. A: Typical steering signal including lane changes from right to left and left to right. The dash lines indicate the threshold for extracting reaction times during the lane change events. B: Steering profiles for all subjects. Individual lines correspond to the average across trials that have the same initial distance to the obstacle. Traces are color coded so that darker colors indicate larger distances. C: Histogram of the reaction time for all subjects.

with respect to the obstacle appearance $(t=0)$. The PSD for each trial was referenced to a baseline defined as the average activity in the time window [-375 0] ms.

\section{RESULtS}

\section{A. Behavior analysis}

A typical trace of the steering signal is shown in Figure 3.A. Values are close to zero when the vehicle is moving straight with clear spikes indicating lane changes during the experiments. The car moves either left (negative) or right (positive) to avoid the obstacle, and afterwards the steering value returns zero to continue moving straight.

Figure 3.B shows the driver steering profiles after obstacle appearance for all subjects. Trials were colored from shorter to larger distances using light to dark tones, respectively. Unsurprisingly, trials in which the obstacle appeared at shorter distances (light traces) exhibit a faster reaction time and larger steering amplitude than those with farther obstacles.

Based on these results we set the steering threshold to estimate the reaction time to the value of 0.01 . The distribution of the reaction time is shown in Figure 3.B. The median response time for all the trials is $0.496 \mathrm{~s}$, and the mean value ( \pm standard deviation) is $0.519 \mathrm{~s} \pm 0.109 \mathrm{~s}$. For the EEG analysis we discarded the extremes of the distribution, corresponding to trials with reaction times smaller than $400 \mathrm{~ms}$ or larger than $650 \mathrm{~ms}$, amounting to $6.88 \%$ of all the recorded trials.

\section{B. ERP analysis}

Event-related potentials (ERPs) of channels $\mathrm{FCz}$ and $\mathrm{CPz}$ are shown in Figures 4.A and B, respectively. The reaction time of the trials is indicated by the darkness of the curves, the darker the faster. Each curve indicates the averaged ERP across all subjects based on the reaction time, i.e., from $0.4 \mathrm{~s}$ to $0.65 \mathrm{~s}$ after the presence of obstacles.

$\mathrm{FCz}$ electrode shows a positive peak at about $270 \mathrm{~ms}$ followed by a negative peak at about $515 \mathrm{~ms}$. Slower trials (light traces) appear to have later peaks than the faster ones. A more marked pattern appears in channel $\mathrm{CPz}$, where a large negative deflection appears at about $300 \mathrm{~ms}$. As before, the peak latency of this component seems modulated by the driver's reaction time. Significant correlation between the peak latency of the early peak -appearing before action onset- and the driver's reaction time was found for both channels. The latency of the positive peak in $\mathrm{FCz}$ have a correlation coefficient of $0.427\left(p<10^{-4}\right)$, while the negative peak in $\mathrm{CPz}$ yields a correlation of $0.683\left(p<10^{-13}\right)$. The $p$ value was obtained using Student's t-distribution with the assumption of bivariate normal distribution.

Consistently with the ERPs, scalp-wide grand average activity at $300 \mathrm{~ms}$ shows strong negative modulation in parietal areas, with a broader positivity over frontal areas, as shown in Figure 4.E. Similarly, figure 4.F shows the correlation between the peak latency of the early ERP component (prior to $400 \mathrm{~ms}$ ) for each electrode. The results show positive correlations in both parietal and frontal regions, with highest correlation value in the former area. These spatial specific correlation patterns suggest that these sites are modulated in the stimulus-driven reaction and are informative about the behavioral reaction time.

\section{Power spectral density}

The grand average of the PSD in $\mathrm{FCz}$ and $\mathrm{CPz}$ are shown in Figure 5.A. We found no evident modulation in the first $200 \mathrm{~ms}$ after the obstacle appearance. In contrast, both electrodes show increased theta power $(4-8 \mathrm{~Hz})$ after this period. Typically, this increase in power centers at about $300 \mathrm{~ms}$ and disappears before the steering onset (i.e. after $400 \mathrm{~ms}$ ). A decrease in the beta power $(20-35 \mathrm{~Hz})$ is also clear in these two electrodes, appearing at about $300 \mathrm{~ms}$, continuing during lane change behavior. In addition, a late increase at about $500 \mathrm{~ms}$ can be observed in the low frequency $(1-4 \mathrm{~Hz})$ activity in FCz. This pattern is not obvious in $\mathrm{CPz}$.

The correlation between the band power (theta and beta) and the reaction time of the steering are analyzed as well, which is similar as reported in the section of ERP analysis. The average band power in the time window between $200 \mathrm{~ms}$ to $400 \mathrm{~ms}$ was computed and used to obtain the correlation coefficient between the reaction time. 

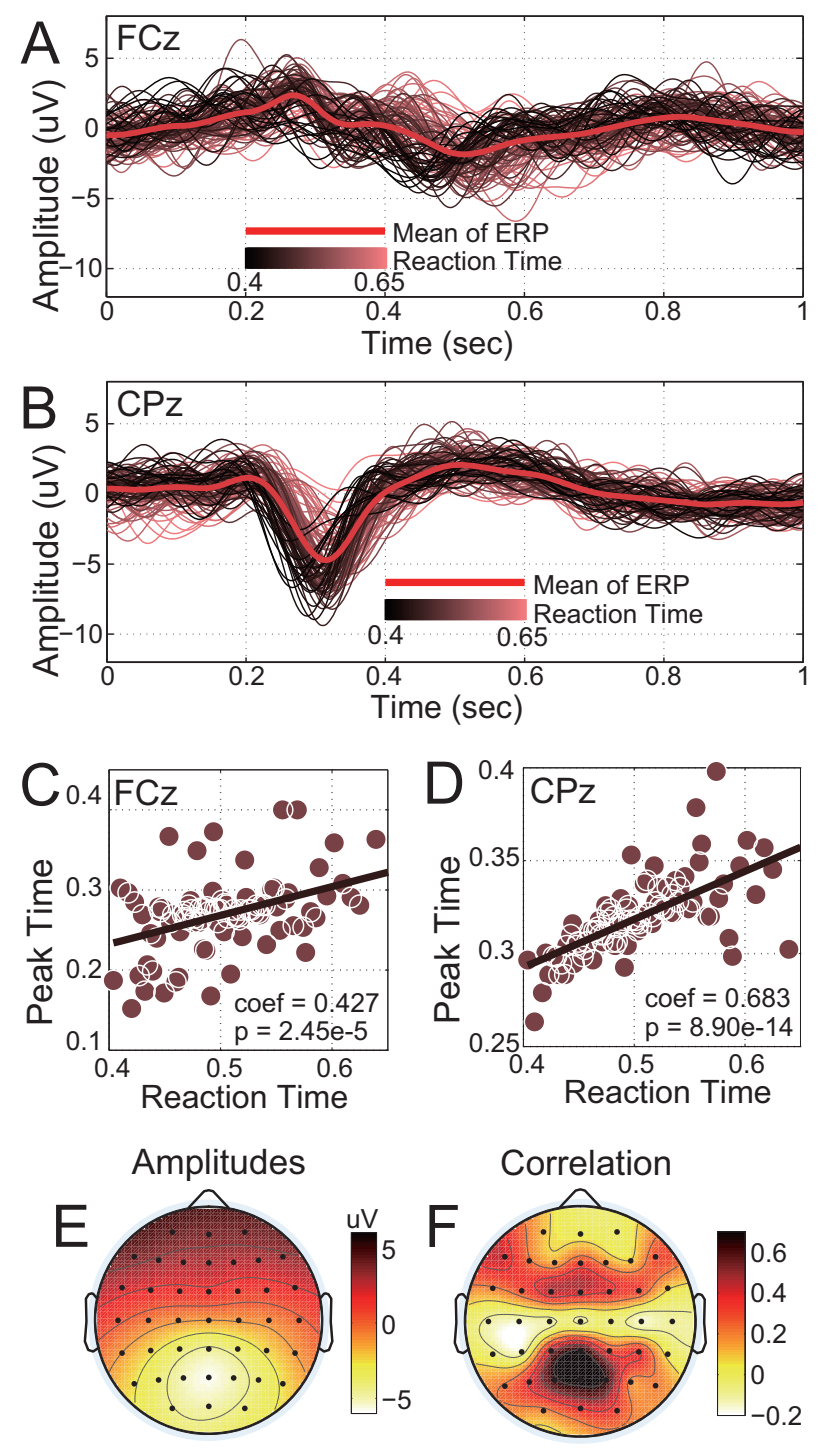

Fig. 4. Event-related potentials and their correlation with reaction time. A and B: ERPs in electrodes FCz and $\mathrm{CPz}$, respectively. Reaction time for each trace is color-coded with darker colors indicating shorter trials. The grand average is shown by the thick red curve. C and D: Correlation coefficients between reaction time and the latency of the early ERP peak in electrodes $\mathrm{FCz}$ and CPz. E: Topography of ERP amplitude for all trials at $300 \mathrm{~ms}$. F: Topographical representation of the correlation coefficients between ERP peak latency and reaction time.

Topographical analysis of spectral modulations for the theta and beta bands is shown in Figure 5.C. Each plot shows the average band power in the window from $200 \mathrm{~ms}$ to $400 \mathrm{~ms}$. The theta power increase is stronger at parietal areas, peaking at $\mathrm{CPz}$ and $\mathrm{Pz}$, also appearing in frontocentral and frontolateral regions. The pattern for the beta band shows broader modulations, particularly in parietal regions. The medial central (electrode $\mathrm{Cz}$ ) and lateral frontal regions do not show evident beta modulation.

The reaction time and the power modulation in the theta


Fig. 5. Spectral analysis. A: PSD of electrodes $\mathrm{FCz}$ and $\mathrm{CPz}$ upon appearance of the obstacle. $\mathrm{B}$ : Correlation between the reaction time and the band power in the theta $(4-8 \mathrm{~Hz})$ and beta $(25-30 \mathrm{~Hz})$ bands for $\mathrm{FCz}$ and $\mathrm{CPz}$. C: Topographies of the average theta power and beta power in the window [200 400] ms. D: Correlation coefficient for theta power and beta power for the whole montage. 
band is significantly correlated, with correlation coefficient -0.3 in $\mathrm{FCz}(p<0.005)$ and -0.34 in $\mathrm{CPz}(p<0.001)$. The correlation coefficients in theta are negative, which indicate that the trials with faster reaction are accompanying with stronger increase in theta. The beta power is positively correlated with the reaction time (the correlation coefficient is 0.2 and 0.18 for $\mathrm{FCz}$ and $\mathrm{CPz}$ respectively), i.e., the faster reaction the more decrease in beta, however, these correlations is not statistically significant $(\mathrm{p}>0.05)$. In the topography of correlation coefficients, we observe that both frontocentral (except $\mathrm{Cz}$ ) and parietal regions are highly correlated with reaction time. The coefficients in frontolateral regions are close to 0.2 , but much lower than frontocentral and parietal regions. In the beta band, the most correlated regions are also frontal and parietal.

\section{DisCUSSION AND CONCLUSION}

This study provides evidence of reliable neural markers of the driver's response variability. In agreement with previous studies performed in simpler experimental protocols [4], these correlations can be observed in parietal and frontal areas. We found significant correlation between the driver's behavioral response and the brain activity prior to the steering action, at the level of the ERP peak latency and power modulations in the theta band.

In our experiments, the characteristic of the external stimuli is varied from trial to trial, due to the distance between the vehicle and the appearing obstacle. The shorter distance leads to higher demand of lane change, not only from the visual perception of closer object (larger) but also the higher temporal urgency of the task, which needs to be reacted more rapidly [11], [12], [13], [14], [15]. Current debates exist about the mechanism of the reduction of response latency in urgent situations, either caused by different CNS pathways or a common pathway but more rapid response latency [13], [14]. The brain patterns obtained in the present study are similar among the reactive trials no matter the diversity of reaction times, in terms of ERP peaks and oscillatory power modulations, given the truth that all responding trials require action within certain period, i.e., the vehicle will be damaged if no response is taken place. Thus our results prove that the brain patterns keep the same among fast and slow reactions but the intensity of these patterns are varied and associated to the responding speed. The verification of different CNS pathways in urgent and non-urgent conditions could be further assessed by adding extra conditions, i.e., stimulus-driven lane change but without danger (if it is not executed) and voluntary intended lane change without any external stimulus.

The frontal and parietal regions are activated during attentional tasks in both human subjects and monkeys [16], [17], and particularly the frontal-parietal coherence reflects the transformation from the sensory representation in parietal cortex into the adjusting behavioral responses in frontal regions [18]. The findings in this study show correlation of responding speed in both frontal and parietal regions, i.e., intensive modulation is associated with faster behavior. Recent evidences show earlier and more dominant neural association in parietal in such exogenous processing comparing with frontal area [19], [20]. Future studies will explore the causal influence between different frontal and parietal regions to find the information flows that are correlated with the reaction time, given the advantage of high temporal resolution of EEG data.

Concluding, the current findings complement recent studies that have identified correlates of other cognitive processes in realistic driving, including drowsiness [6], [21], [22], [23], emergency braking [9], [24], error-awareness [25], anticipation of self-motivated steering [8] and braking actions [7], as well as visual attention [26]. We purport that future driving assistive systems can exploit information derived from these signals -decoded through a brain-machine interface system-, in combination with information from in-car sensors to tailor the support they provide both to the perceived conditions of the environment as well as the internal state of the driver [27].

\section{ACKNOWLEDGMENT}

This study was supported by Nissan Motor Co. Ltd., and carried out under the "Research on Brain Machine Interface for Drivers" project. This paper only reflects the authors' view and the funding agencies are not liable for any use that may be made of the information contained herein.

\section{REFERENCES}

[1] F. C. Donders, "On the speed of mental processes," Acta psychologica, vol. 30, pp. 412-431, 1969.

[2] A. N. Carlsen, R. Chua, J. T. Inglis, D. J. Sanderson, and I. M. Franks, "Can prepared responses be stored subcortically?" Experimental brain research, vol. 159, no. 3, pp. 301-309, 2004.

[3] S. Kastner and L. G. Ungerleider, "Mechanisms of visual attention in the human cortex." Annu Rev Neurosci, vol. 23, pp. 315-341, 2000. [Online]. Available: http://dx.doi.org/10.1146/annurev.neuro.23.1.315

[4] A. D. Gerson, L. C. Parra, and P. Sajda, "Cortical origins of response time variability during rapid discrimination of visual objects." Neuroimage, vol. 28, no. 2, pp. 342-353, Nov 2005. [Online]. Available: http://dx.doi.org/10.1016/j.neuroimage.2005.06.026

[5] T. J. Buschman and E. K. Miller, "Top-down versus bottom-up control of attention in the prefrontal and posterior parietal cortices." Science, vol. 315, no. 5820, pp. 1860-1862, Mar 2007. [Online]. Available: http://dx.doi.org/10.1126/science.1138071

[6] C.-T. Lin, L.-W. Ko, and T.-K. Sheng, "Computational intelligent brain computer interaction and its applications on driving cognition," IEEE Computational Intelligence Magazine, vol. 4, no. 4, pp. 32-46, 2009.

[7] Z. Khaliliardali, R. Chavarriaga, L. A. Gheorghe, and J. d. R. Millán, "Detection of anticipatory brain potentials during car driving." Conf Proc IEEE Eng Med Biol Soc, vol. 2012, pp. 3829-3832, 2012. [Online]. Available: http://dx.doi.org/10.1109/EMBC.2012.6346802

[8] L. A. Gheorghe, R. Chavarriaga, and J. d. R. Millán, "Steering Timing Prediction in a Driving Simulator Task," in Proceedings of the 35th Annual International Conference of the IEEE Engineering in Medicine and Biology Society, 2013, pp. 6913-6916.

[9] S. Haufe, M. S. Treder, M. F. Gugler, M. Sagebaum, G. Curio, and B. Blankertz, "EEG potentials predict upcoming emergency brakings during simulated driving." J Neural Eng, vol. 8, no. 5, p. 056001, Jul 2011. [Online]. Available: http://dx.doi.org/10.1088/17412560/8/5/056001

[10] G. Borghini, L. Astolfi, G. Vecchiato, D. Mattia, and F. Babiloni, "Measuring neurophysiological signals in aircraft pilots and car drivers for the assessment of mental workload, fatigue and drowsiness." Neurosci Biobehav Rev, Oct 2012. [Online]. Available: http://dx.doi.org/10.1016/j.neubiorev.2012.10.003

[11] D. Pins and C. Bonnet, "On the relation between stimulus intensity and processing time: Pirons law and choice reaction time," Perception and Psychophysics, vol. 58, no. 3, pp. 390-400, 1996. [Online]. Available: http://dx.doi.org/10.3758/BF03206815 
[12] A. Bell, M. Meredith, A. Van Opstal, and D. Munoz, "Stimulus intensity modifies saccadic reaction time and visual response latency in the superior colliculus," Experimental Brain Research, vol. 174, no. 1, pp. 53-59, 2006. [Online]. Available: http://dx.doi.org/10.1007/s00221006-0420-Z

[13] W. Gage, K. Zabjek, S. Hill, and W. McIlroy, "Parallels in control of voluntary and perturbation-evoked reach-to-grasp movements: Emg and kinematics," Experimental Brain Research, vol. 181, no. 4, pp. 627-637, 2007. [Online]. Available: http://dx.doi.org/10.1007/s00221-007-0959-3

[14] B. Lakhani, K. V. Ooteghem, V. Miyasike-daSilva, S. Akram, A. Mansfield, and W. E. McIlroy, "Does the movement matter? determinants of the latency of temporally urgent motor reactions," Brain Research, vol. 1416, no. 0, pp. 35 - 43, 2011. [Online]. Available: http://www.sciencedirect.com/science/article/pii/S0006899311014739

[15] B. Lakhani, A. H. Vette, A. Mansfield, V. Miyasike-daSilva, and W. E. McIlroy, "Electrophysiological correlates of changes in reaction time based on stimulus intensity," PLOS ONE, vol. 7, 052012.

[16] G. Buzski, "Theta rhythm of navigation: Link between path integration and landmark navigation, episodic and semantic memory," Hippocampus, vol. 15, no. 7, pp. 827-840, 2005. [Online]. Available: http://dx.doi.org/10.1002/hipo.20113

[17] G. G. Gregoriou, S. J. Gotts, H. Zhou, and R. Desimone, "Long-range neural coupling through synchronization with attention," in Attention, ser. Progress in Brain Research, N. Srinivasan, Ed. Elsevier, 2009, vol. 176, pp. 35 - 45. [Online]. Available: http://www.sciencedirect.com/science/article/pii/S0079612309176033

[18] L. Herzog, K. Salehi, K. S. Bohon, and M. C. Wiest, "Pre-stimulu frontal-parietal coherence predicts auditory detection performance in rats," Journal of Neurophysiology, 2014.

[19] T. J. Buschman and E. K. Miller, "Top-down versus bottom-up control of attention in the prefrontal and posterior parietal cortices," Science, vol. 315, no. 5820, pp. 1860-1862, 2007. [Online]. Available: http://www.sciencemag.org/content/315/5820/1860.abstract

[20] F. Arcizet, K. Mirpour, and J. W. Bisley, "A pure salience response in posterior parietal cortex," Cerebral Cortex, vol. 21, no. 11, pp. 2498-2506, 2011. [Online]. Available: http://cercor.oxfordjournals.org/content/21/11/2498.abstract
[21] C.-T. Lin, S.-A. Chen, T.-T. Chiu, H.-Z. Lin, and L.-W. Ko, "Spatial and temporal EEG dynamics of dual-task driving performance." J Neuroeng Rehabil, vol. 8, p. 11, 2011. [Online]. Available: http://dx.doi.org/10.1186/1743-0003-8-11

[22] R. N. Khushaba, S. Kodagoda, S. Lal, and G. Dissanayake, "Driver drowsiness classification using fuzzy wavelet-packetbased feature-extraction algorithm." IEEE Trans Biomed Eng, vol. 58, no. 1, pp. 121-131, Jan 2011. [Online]. Available: http://dx.doi.org/10.1109/TBME.2010.2077291

[23] C.-H. Chuang, L.-W. Ko, T.-P. Jung, and C.-T. Lin, "Kinesthesia in a sustained-attention driving task." Neuroimage, vol. 91, pp. 187-202, May 2014. [Online]. Available: http://dx.doi.org/10.1016/j.neuroimage.2014.01.015

[24] S. Haufe, J.-W. Kim, I.-H. Kim, A. Sonnleitner, M. Schrauf, G. Curio, and B. Blankertz, "Electrophysiology-based detection of emergency braking intention in real-world driving." J Neural Eng, vol. 11, no. 5, p. 056011, Aug 2014. [Online]. Available: http://dx.doi.org/10.1088/1741$2560 / 11 / 5 / 056011$

[25] H. Zhang, R. Chavarriaga, L. A. Gheorghe, and J. d. R. $\mathrm{R}$ Millán, "Inferring driver's turning direction through detection of error related brain activity." Conf Proc IEEE Eng Med Biol Soc, vol. 2013, pp. 2196-2199, Jul 2013. [Online]. Available: http://dx.doi.org/10.1109/EMBC.2013.6609971

[26] H. Renold, R. Chavarriaga, L. A. Gheorghe, and J. d. R. Millán, "EEG correlates of active visual search during simulated driving: An exploratory study," in IEEE International Conference on Systems, Man, and Cybernetics, San Diego, USA, 2014.

[27] S. Saeedi, T. Carlson, R. Chavarriaga, and J. d. R. Millán, "Making the most of context-awareness in brain-computer interfaces," in IEEE International Conference on Cybernetics, 2013. 